\title{
Effect of Thalidomide on Endotoxin-Induced Decreases in Activity and Expression of Hepatic Cytochrome P450 3A2
}

\author{
Jun UeYama,${ }^{a, b}$ Masayuki NadaI,${ }^{c}$ Ying Lan Zhao, ${ }^{d, e}$ Hiroaki Kanazawa,${ }^{f}$ Kenji Takagi, ${ }^{a}$ \\ Takaaki Kondo, ${ }^{a}$ Kenzo Takagi, ${ }^{a}$ Shinya Wakusawa, ${ }^{a}$ Fumie Abe, ${ }^{e}$ Hiroko Saito, ${ }^{e}$ \\ Ken-ichi MiYamoto, ${ }^{b}$ and Takaaki HaSEgaWA ${ }^{* e}$ \\ ${ }^{a}$ Department of Medical Technology, Nagoya University School of Health Sciences; 1-1-20 Daikominami, Higashi-ku, \\ Nagoya 461-8673, Japan: ${ }^{b}$ Department of Pharmacy, Kanazawa University Hospital; 13-1 Takara-machi, Kanazawa \\ 920-8641, Japan: ${ }^{C}$ Faculty of Pharmacy, Meijo University; 150 Yagotoyama, Tenpaku-ku, Nagoya 468-8503, Japan: \\ ${ }^{d}$ State Key Laboratory of Biotherapy and Cancer Center, West China Hospital, West China Medical School, Sichuan \\ University; Chengdu 610041, China: ${ }^{e}$ Department of Pharmacy and Pharmacokinetics, Aichi Medical University School of \\ Medicine; 21 Nagakute-cho, Aichi 480-1195, Japan: and ${ }^{f}$ School of Nursing, University of Shizuoka; 52-1 Yada, Suruga- \\ ku, Shizuoka 422-8526, Japan. Received March 19, 2008; accepted May 15, 2008; published online May 19, 2008
}

Thalidomide has been reported to inhibit the production of tumor necrosis factor-alpha (TNF- $\alpha$ ) and nitric oxide (NO) that are involved in the down-regulation of hepatic cytochrome P450 (CYP) induced by endotoxin. In the present study, we investigated the effects of thalidomide on endotoxin-induced decreases in the activity and expression of hepatic CYP3A2 in rats. Thalidomide $(50 \mathrm{mg} / \mathrm{kg})$ was administered orally $22 \mathrm{~h}$ and $2 \mathrm{~h}$ before intraperitoneal injection of endotoxin $(1 \mathrm{mg} / \mathrm{kg})$. Twenty-four hours after the injection of endotoxin, antipyrine clearance experiments were conducted, in which the rats were sacrificed and protein levels of hepatic CYP3A2 were measured. There were no significant differences in the histopathological changes in the liver between the endotoxin-treated and endotoxin plus thalidomide-treated rats. Thalidomide had no effect on the systemic clearance of antipyrine, which is a proper indicator for hepatic CYP3A2 activity, whereas it enhanced endotoxin-induced decrease in the systemic clearance of antipyrine. Western blot analysis revealed that thalidomide had no effect on the protein levels of hepatic CYP3A2, whereas it enhanced the down-regulation of hepatic CYP3A2 by endotoxin. However, there were no significant differences in the concentrations of TNF- $\alpha$ and NO in plasma between the endotoxin-treated and endotoxin plus thalidomide-treated rats. The present findings suggest that thalidomide enhances endotoxin-induced decreases in the activity and expression of hepatic CYP3A2.

Key words thalidomide; endotoxin; cytochrome P450 3A2; nitric oxide; tumor necrosis factor-alpha

Inflammatory cytokines induced by endotoxin, which is an active component in the outer membrane of gram-negative bacteria, are known to induce damages in numerous organs including the liver. The liver plays a pivotal role for the detoxification and elimination of endogenous and exogenous toxic compounds. Other investigators and we have reported that endotoxin and/or endotoxin-induced inflammatory mediators reduce hepatic drug-metabolizing enzyme activity by reducing the cytochrome $\mathrm{P} 450$ (CYP) activity and expression of the mRNA and protein. ${ }^{1-5)}$ Minamiyama and colleagues ${ }^{1)}$ have reported that the overproduction of nitric oxide (NO) in plasma by endotoxin directly inactivates hepatic CYP activities. Moreover, Hara and Adachi ${ }^{6}$ ) have reported that NO down-regulates CYP gene expression by directly inhibiting hepatic nuclear factor-4 (HNF4), which is an important factor for constitutive expression of CYP. These findings suggest that NO plays an important role in the decreased hepatic CYP activity and/or protein in endotoxemia.

There are a number of reports suggesting that inflammatory cytokines, including tumor necrosis factor-alpha (TNF$\alpha$ ), interleukin- $1 \beta$ (IL-1 $\beta$ ), IL-6 and interferon, might play an important role in the down-regulation of hepatic CYP isoforms induced by endotoxin. ${ }^{3,7-9)}$ Additionally, it is proposed that one possible mechanism of the down-regulation of CYP isoforms induced by endotoxin is closely associated with the repression of nuclear hormone receptors constitutive androstane receptor (CAR), pregnane $\mathrm{X}$ receptor (PXR) and peroxisome proliferator-activated receptor- $\gamma$ (PPAR- $\gamma$ ), which are the key regulators of some CYP subtype gene ex- pressions in the liver. ${ }^{4,10)}$

It has recently been found that thalidomide, initially used as a sedative and hypnotic, has anti-inflammatory, immunomodulatory, and anti-angiogenic effects. ${ }^{11,12}$ ) Therefore, it is newly used in the treatment of various diseases, including multiple myeloma, cancer, and others. ${ }^{13-16)}$ Although the mechanism responsible for clinical efficacy of thalidomide is still not clear, several investigators have suggested the possible involvement of selective inhibition of TNF- $\alpha$ production. ${ }^{17-21)}$ There are interesting reports suggesting that thalidomide improves endotoxin- or alcohol-induced liver injury, ${ }^{22,23)}$ and prolongs the survival rate following the experimental sepsis induced by endotoxin or bacterial challenge in rats. $^{24,25)}$ Shimazawa et al. $^{26)}$ have reported that thalidomide and its analogs have NO synthase (NOS)-inhibitory activity in vitro, but the activity of thalidomide is weak. Enomoto et $a{ }^{27)}$ reported that thalidomide abolishes the endotoxin-induced increase in CD14 expression in Kupffer cells in vitro. To our knowledge, the effect of thalidomide on the reduction in hepatic function in endotoxemia has not yet been elucidated.

The purpose of the present study was to investigate the effect of thalidomide on endotoxin-induced decreases in hepatic function, particularly CYP-mediated drug-metabolizing enzyme activity and expression of CYP3A2 which is a major CYP isoform in rats ${ }^{28)}$ and plays a key role in phase I detoxification of endogenous and exogenous compounds. 


\section{MATERIALS AND METHODS}

Chemicals Endotoxin was prepared from a clinically isolated Klebsiella pneumoniae LEN-1 $\left(\mathrm{O} 3: \mathrm{K}^{-}{ }^{-}\right)$, which was identical to that used in the previous studies. ${ }^{4,5)}$ Endotoxin was dissolved in saline at a concentration of $0.5 \mathrm{mg} / \mathrm{ml}$. (+)Thalidomide, (-)-thalidomide, ( \pm )-thalidomide and antipyrine were purchased from Sigma Chemicals (St. Louis, MO, U.S.A.). All other reagents were obtained commercially and of the highest purity available. $( \pm)$-Thalidomide used in the in vivo experiments was suspended in $0.9 \%$ carboxymethylcellulose (CMC).

Animals Nine-week-old male Wistar rats (weighing $290-310 \mathrm{~g}$ ) used in this study were supplied by Charles River Japan (Yokohama, Japan). The animals were housed under the standardized conditions (room temperature approximately $25^{\circ} \mathrm{C}$, humidity $55 \pm 5 \%, 12 \mathrm{~h}$ light: $12 \mathrm{~h}$ dark schedule) with commercial food and water freely available. The procedures involving animals and their care conformed to the international guidelines, Principles of Laboratory Animal Care (NIH publication No. 85-23, revised 1985) and Guiding Principles for the Care and Use of Laboratory Animals of Nagoya University, Japan.

Experimental Protocols Eighteen hours before endotoxin administration, rats were anesthetized by sodium pentobarbital ( $20 \mathrm{mg} / \mathrm{kg}$ of body weight), and the right jugular vein and peritoneal cavity were cannulated with polyethylene tubes (Natsume PE45, Tokyo, Japan) for blood collection and drug administration. To evaluate the effect of thalidomide on endotoxin-induced hepatic function, rats received thalidomide $(50 \mathrm{mg} / \mathrm{kg}$ orally) $22 \mathrm{~h}$ and $2 \mathrm{~h}$ before endotoxin injection $(1 \mathrm{mg} / \mathrm{kg}$ intraperitoneally). Control rats received an equivalent volume of $\mathrm{CMC}$ solution or saline in place of thalidomide or endotoxin. The dose of thalidomide used in this study was chosen on the basis of that used in previous studies. $^{25)}$ Twenty-four hours after injection of endotoxin or saline, antipyrine clearance experiments were conducted, since we previously reported that the level of endotoxin-induced decrease in hepatic drug-metabolizing enzyme activity reaches the maximum $24 \mathrm{~h}$ after endotoxin administration. ${ }^{29}$ ) Rats received a bolus intravenous injection of antipyrine ( $20 \mathrm{mg} / \mathrm{kg}$ of body weight), which was dissolved in saline at the concentration of $10 \mathrm{mg} / \mathrm{ml}$. Blood samples were collected at designated intervals $(30,60,90,120,180,240,300 \mathrm{~min}$ after antipyrine injection). Plasma samples were obtained by centrifugation of blood samples at $1200 \times \boldsymbol{g}$ for $5 \mathrm{~min}$ at $4{ }^{\circ} \mathrm{C}$ and were stored at $-40{ }^{\circ} \mathrm{C}$ until analysis.

Biochemical Determinations Concentrations of nitrate/nitrite $(\mathrm{NOx})$ in plasma were measured with a commercial kit (Nitrate/Nitrite Colorimetric Assay Kit, Cayman Chemical, Ann Arbor, MI, U.S.A.). Briefly, the plasma samples obtained from the in vivo experiments were ultrafiltered (molecular cut-off of 10000 ) at $6000 \times \boldsymbol{g}$ for $60 \mathrm{~min}$ at $4{ }^{\circ} \mathrm{C}$. The ultrafiltrate was allowed to incubate for $3 \mathrm{~h}$ with nitrate reductase and its cofactor and to react with Griess reagents for $20 \mathrm{~min}$. Absorbance was measured at $540 \mathrm{~nm}$ with a microplate reader (Molecular Devices, Crawley, U.K.). The within-day and between-day coefficients of variation for this assay were less than $5 \%$ at the concentration of $50 \mu \mathrm{M}$.

Concentrations of albumin and total protein in plasma were determined using the well-known bromcresol green and biuret reactions, respectively. The activity of aspartate aminotransferase (AST) was measured with a commercial kit (Wako, Tokyo, Japan).

To measure the concentration of TNF- $\alpha$ in plasma, blood samples were taken $2 \mathrm{~h}$ after injection of endotoxin. Concentration of TNF- $\alpha$ was measured with a commercial kit (Endogen ELISA kit, Pierce Biotechnology, Inc., Rockford, IL, U.S.A.) according to the manufacturer's instructions.

Drug Analysis Concentrations of antipyrine and thalidomide in plasma were measured by high-performance liquid chromatography (HPLC). The apparatus used for HPLC was a Shimadzu LC-10A system (Kyoto, Japan) consisting of an LC-10A liquid pump and an autoinjector (SPD-10 AV), equipped with a UV-VIS detector (SPD-10 AV). The concentrations of antipyrine in plasma were determined as described above. ${ }^{4)}$ The assay conditions for both (+)-thalidomide and $(-)$-thalidomide were as follows: column, CHIRALPAK AD-H $(0.46 \times 250 \mathrm{~mm})$ (Daicel Chemical Industries, Tokyo, Japan); mobile phase, methanol; wavelength, $220 \mathrm{~nm}$; temperature, $25^{\circ} \mathrm{C}$; and flow rate, $1.5 \mathrm{ml} / \mathrm{min}$. For measurement of both (+)-thalidomide and ( - )-thalidomide, $50 \mu \mathrm{l}$ of each sample and $200 \mu \mathrm{l}$ of acetonitrile were mixed and centrifuged at $6000 \times \boldsymbol{g}$ for $5 \mathrm{~min}$. After centrifugation, the supernatant $(200 \mu \mathrm{l})$ was evaporated at $45^{\circ} \mathrm{C}$ to dryness with a gentle nitrogen stream. The residue was dissolved in $200 \mu \mathrm{l}$ of the mobile phase, and $100 \mu \mathrm{l}$ of it was injected for HPLC analysis. This assay was shown to be linear for the concentration studied, with a correlation coefficient of 0.999 . The detection limit for $(+)$-thalidomide and $(-)$-thalidomide was $0.05 \mu \mathrm{g} / \mathrm{ml}$.

Western Blot Analysis The microsomes were prepared according to the prior published methods ${ }^{30)}$ with some modifications. Briefly, liver (approximately $1 \mathrm{~g}$ ) was homogenized at $4{ }^{\circ} \mathrm{C}$ with a Teflon homogenizer using $1.15 \% \mathrm{KCl}$. The homogenate was centrifuged at $12000 \times \boldsymbol{g}$ for $25 \mathrm{~min}$ at $4{ }^{\circ} \mathrm{C}$. The supernatant was further centrifuged at $100000 \times \boldsymbol{g}$ for $90 \mathrm{~min}$ at $4{ }^{\circ} \mathrm{C}$ to obtain the microsomal fraction. The pellet obtained was suspended in $1.15 \% \mathrm{KCl}$.

Western blot analysis of CYP3A2 was performed according to the methods described above. $\left.{ }^{4,5}\right)$ The protein concentration of the microsomal fraction was measured by Bio-Rad Protein Assay (Bio-Rad Laboratories, Richmond, CA, U.S.A.), using bovine serum albumin (Sigma Chemicals) as a standard. One microgram of protein was separated by electrophoresis on a $10 \%$ polyacrylamide gel containing $0.1 \%$ sodium dodecyl sulfate (SDS) and then transferred to a polyvinylidene difluoride (PVDF) membrane (Millipore, Bedford, MA, U.S.A.). The membrane was blocked in phosphate-buffered saline (PBS) containing 0.1\% Tween-20 and $4 \%$ nonfat dry milk for $30 \mathrm{~min}$ at room temperature. The membrane was incubated with rabbit polyclonal antibody (Daiichi Pure Chemicals, Tokyo, Japan) to rat CYP3A2, washed, and incubated with horseradish peroxidase-labeled anti-rabbit immunoglobulin G (Amersham Biosciences, Piscataway, NJ, U.S.A.), and washed. The immune complexes were visualized using the enhanced chemiluminescence detection system (ECL, GE Healthcare U.K. Ltd., Buckinghamshire, U.K.).

To quantify the relative levels of CYP3A2 in each membrane, the intensity of the stained bands was estimated by the NIH image program (Bethesda, MD, U.S.A.). 
Data Analysis The concentration-time data for antipyrine in each rat were analyzed individually using a noncompartmental model. The area under the concentrationtime curve $(A U C)$ and the area under the first-moment curve $(A U M C)$ were calculated by the trapezoidal method up to the last measured plasma concentration and were extrapolated to infinity. Systemic clearance $\left(C L_{\mathrm{SYS}}\right)$ was calculated by dividing the dose by the $A U C$. The steady state volume of distribution $\left(V_{\mathrm{SS}}\right)$ was calculated as $V_{\mathrm{SS}}=C L_{\mathrm{SYS}} \times M R T$, using the equation $M R T=A U M C / A U C$ where $M R T$ represents the mean residence time.

Statistical Analysis All data are expressed as means S.E.M. One-way analysis of variance (ANOVA) and Fisher's LSD test were used to determine the statistical significance of differences among experimental groups. The 0.05 level of probability was used as the criterion of significance. These statistical analyses were performed using StatView software (Abacus Concepts, Berkeley, CA, U.S.A.).

\section{RESULTS}

Biochemical and Histopathological Examinations We measured concentrations of (+)-thalidomide and (-)thalidomide in plasma just before the injection of endotoxin in rats. No significant differences in the plasma concentrations were observed between $(+)$-thalidomide $(3.3 \pm$ $0.4 \mu \mathrm{g} / \mathrm{ml}$ ) and (-)-thalidomide $(3.6 \pm 0.4 \mu \mathrm{g} / \mathrm{ml})$. At the dose of thalidomide used in this study, it was possible to obtain the concentration which inhibits the production of TNF$\alpha$ induced by endotoxin. ${ }^{31)}$ Biochemical data such as concentrations of total protein, albumin and AST in plasma are summarized in Table 1. As shown in Table 1, endotoxin sig-

Table 1. Biochemical Data for Endotoxin-, Endotoxin Plus Thalidomideand Thalidomide-Treated and Untreated Rats

\begin{tabular}{lccc}
\hline \multicolumn{1}{c}{ Treatment } & Albumin $(\mathrm{g} / \mathrm{dl})$ & Total protein $(\mathrm{g} / \mathrm{dl})$ & AST $(\mathrm{IU} / \mathrm{l})$ \\
\hline Untreated & $2.23 \pm 0.03$ & $4.74 \pm 0.25$ & $108 \pm 8.00$ \\
Endotoxin & $1.87 \pm 0.09^{a)}$ & $4.81 \pm 0.22$ & $122 \pm 19.0$ \\
Endotoxin plus & $2.06 \pm 0.08$ & $4.83 \pm 0.18$ & $111 \pm 7.40$ \\
Thalidomide & & & \\
Thalidomide & $2.19 \pm 0.08$ & $5.34 \pm 0.28$ & $84 \pm 6.30^{b)}$
\end{tabular}

Thalidomide $(50 \mathrm{mg} / \mathrm{kg})$ was administered orally $22 \mathrm{~h}$ and $2 \mathrm{~h}$ before injection of endotoxin $(1 \mathrm{mg} / \mathrm{kg})$ or saline. Blood samples were collected $24 \mathrm{~h}$ after injection of endotoxin. Each value represents the mean \pm S.E.M. $(n=4-5) . a)$ and $b)$ significantly different from untreated and endotoxin, respectively $(p<0.05)$.

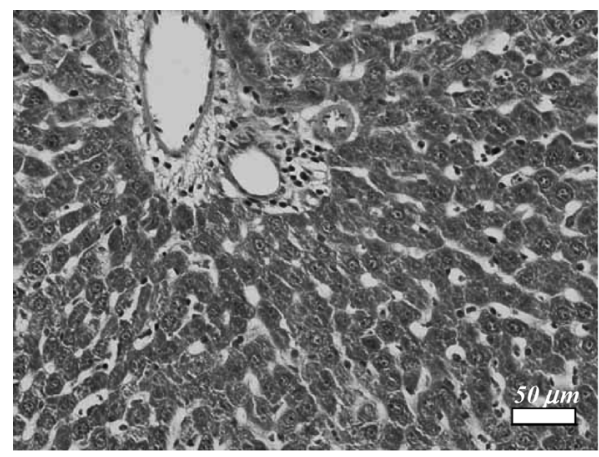

Endotoxin alone nificantly decreased the levels of albumin in plasma, but not total protein or the activity of AST, $24 \mathrm{~h}$ after injection. Pretreatment with thalidomide protected endotoxin-induced decrease in the levels of albumin in plasma. However, thalidomide significantly, but slightly, decreased the activity of AST in plasma.

We previously reported that the dose of endotoxin used in this study did not induce any evidence of massive necrotic and apoptotic areas in rat liver tissue. ${ }^{4)}$ As shown in Fig. 1, histopathological examinations revealed that there was no difference in the light microscopy of the liver tissue between endotoxin- and endotoxin plus thalidomide-treated rats. These results suggest that thalidomide does not induce damage to the liver.

Effect of Thalidomide on Endotoxin-Induced Decrease in Hepatic Drug-Metabolizing Enzyme Activity To investigate the effect of thalidomide on endotoxin-induced decrease in hepatic drug-metabolizing enzyme activity, we measured the systemic clearance of antipyrine, which is a proper indicator for hepatic drug-metabolizing enzyme activity. ${ }^{4,5)}$ As shown in Fig. 2, endotoxin significantly decreased the systemic clearance of antipyrine $(0.40 \pm 0.03 \mathrm{l} / \mathrm{h} / \mathrm{kg})$ by approximately $20 \%$ compared with that in untreated rats $(0.52 \pm 0.051 / \mathrm{h} / \mathrm{kg})$. Thalidomide alone had no effect on the systemic clearance of antipyrine $(0.49 \pm 0.05 \mathrm{l} / \mathrm{h} / \mathrm{kg})$, but the systemic clearance of antipyrine was decreased more in endotoxin plus thalidomide-treated rats $(0.29 \pm 0.02 \mathrm{l} / \mathrm{h} / \mathrm{kg})$ than that in endotoxin-treated rats. There were no significant differences in the volume of distribution at steady state $\left(V_{\mathrm{SS}}\right)$ of antipyrine among the treatment groups, indicating that the decreased systemic clearance of antipyrine by endotoxin was

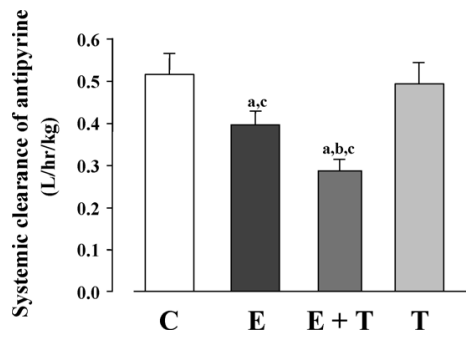

Fig. 2. Effect of Endotoxin and/or Thalidomide on Systemic Clearance of Antipyrine in Rats

Each column represents the mean \pm S.E.M. $(n=3-4)$. C, control; E, endotoxin; T, thalidomide. ${ }^{a}$ Significantly different from untreated. ${ }^{b, c}$ Significantly different from endotoxin and thalidomide, respectively.

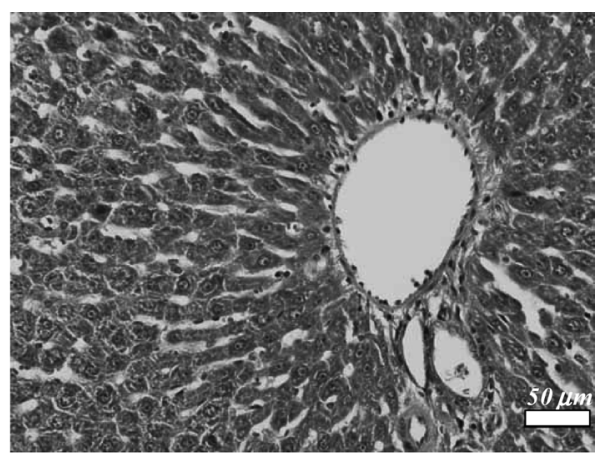

Endotoxin + Thalidomide

Fig. 1. Light Micrograph of Rat Liver Treated with Endotoxin (Left) and Endotoxin Plus Thalidomide (Right) 
caused by alterations in drug-metabolizing enzyme activity.

Effect of Thalidomide on Endotoxin-Induced DownRegulation of Hepatic CYP3A2 The effect of thalidomide on the constitutive expression levels of hepatic CYP3A2 and on endotoxin-induced down-regulation of CYP3A2 was investigated by Western blot analysis. As shown in Fig. 3, endotoxin significantly decreased the protein levels of CYP3A2 to approximately $40 \%$ of that in untreated rats. Thalidomide had no effect on the constitutive expression of hepatic CYP3A2, whereas it further down-regulated endotoxin-induced decrease in the expression of CYP3A2 to approximately $60 \%$ and $25 \%$ of that in endotoxin-treated rats and untreated rats, respectively.

Effect of Thalidomide on Endotoxin-Induced Overproduction of NOx and TNF- $\alpha$ in Plasma Mean plasma concentration-time curves of NOx in endotoxin-, endotoxin plus thalidomide-, thalidomide-treated rats and untreated rats are represented in Fig. 4. Concentrations of $\mathrm{NOx}$ in plasma reached the maximum levels around $8 \mathrm{~h}$ after injection of endotoxin. The $A U C$ of plasma NOx in rats treated with endotoxin was 10-fold larger than that in the untreated rats (approximately $660 \mu \mathrm{M} \cdot \mathrm{h} / \mathrm{ml}$ ). Thalidomide did not change the $A U C$ of NOx in either untreated or endotoxin-treated rats.

To investigate the influence of thalidomide on the production of TNF- $\alpha$ induced by endotoxin, the concentration of TNF- $\alpha$ in plasma $2 \mathrm{~h}$ after injection of endotoxin was measured. The concentration of TNF- $\alpha$ in endotoxin-treated rats was $2215 \pm 527 \mathrm{pg} / \mathrm{ml}$, which was not significantly different

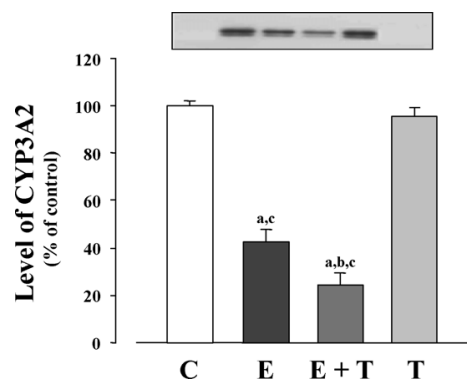

Fig. 3. Effect of Endotoxin and/or Thalidomide on Hepatic CYP3A2 Protein in Rats

Each column represents the mean \pm S.E.M. $(n=3-4)$. C, control; E, endotoxin; T, thalidomide. ${ }^{a}$ Significantly different from untreated. ${ }^{b, c}$ Significantly different from endotoxin and thalidomide, respectively.

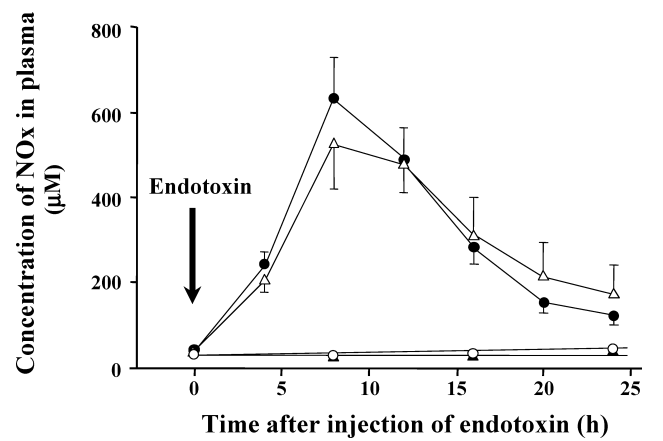

Fig. 4. Effect of Thalidomide on Plasma Concentration-Time Curves of NOx in Rats

Thalidomide $(50 \mathrm{mg} / \mathrm{kg})$ was administered $22 \mathrm{~h}$ and $2 \mathrm{~h}$ before endotoxin injection $(1 \mathrm{mg} / \mathrm{kg})$. Blood samples were collected at designated intervals to measure concentrations of NOx in plasma. Each symbol represents the mean \pm S.E.M. $(n=3-7)$. Symbols, $\mathrm{O}$, control; $\boldsymbol{O}$, endotoxin; $\triangle$, endotoxin plus thalidomide; $\boldsymbol{\Lambda}$, thalidomide alone. from that in endotoxin plus thalidomide-treated rats $(2173 \pm$ $328 \mathrm{pg} / \mathrm{ml})$.

\section{DISCUSSION}

The present study is the first report to demonstrate that thalidomide has effects on the function of detoxication in endotoxemia, although thalidomide is known to have an inhibitory effect against inflammatory states. In the present study, we focused on the effect of thalidomide on the activity and expression of hepatic CYP3A2, which is an important hepatic drug-metabolizing enzyme for physiological function and homeostasis in animals because it catalyzes the oxidation of many kinds of steroids, xenobiotics and drugs, ${ }^{28,32)}$ and is sensitive to some cytokines. ${ }^{2)}$ It is well known that hepatic CYP3A2-dependent drug-metabolizing enzyme activity is decreased by various trigger factors such as drugs, diseases, infection and inflammation.

First, to clarify the effect of thalidomide on the constitutive activity of hepatic drug-metabolizing enzyme and endotoxin-induced decrease in the activity of hepatic drug-metabolizing enzyme, we performed an antipyrine clearance experiment. In this experiment, significant decreases in the systemic clearance of antipyrine were observed in endotoxinand endotoxin plus thalidomide-treated rats without histopathological changes in the liver. The degree of the decreased systemic clearance was significantly greater in endotoxin plus thalidomide-treated rats than that in endotoxin-treated rats. These results suggest that thalidomide decreases the hepatic drug metabolizing enzyme activity more in the presence of endotoxin than in the absence of endotoxin probably due to the alteration of inflammatory response, although the precise mechanism remains unclear at present. Possible mechanisms of decreased hepatic drug metabolizing enzyme activity by endotoxin have been proposed as follows. One of the mechanisms is the down-regulation of $\mathrm{mRNA}$ and protein levels of CYP3A2 which is major enzyme in the rat liver and is related to antipyrine metabolism, by the production of cytokines, ${ }^{2,5,33)}$ however, the precise role of these cytokines in the expression of CYP3A2 remains unclear. The other is the direct inactivation of hepatic CYP3A2 activity by overproduction of NO, which is mainly produced by inducible NOS (iNOS). ${ }^{1,34)}$ In the present study, plasma concentration-time curves of NOx in endotoxin-treated rats were almost the same as those in endotoxin plus thalidomide-treated rats, suggesting that thalidomide does not inhibit endotoxin-induced overproduction of NO in plasma. These results may be supported by the previous studies demonstrating that NOSinhibitory activity of thalidomide in vitro is weak. ${ }^{26)}$

Second, we examined the effects of thalidomide on the constitutive expression and endotoxin-induced down-regulation of CYP3A2 using Western blot analysis. This experiment revealed that thalidomide had no effect on the constitutive expression of CYP3A2, and the degree of the decreased protein levels of CYP3A2 was greater in endotoxin plus thalidomide-treated rats than that in endotoxin-treated rats, which can be agreed well with the results obtained from the antipyrine clearance experiment. It is reported that thalidomide selectively inhibits the production of TNF- $\alpha,{ }^{24,31,35)}$ due to inhibition of endotoxin-induced increase in the intracellular levels of calcium, which plays a crucial role in the pro- 
duction of TNF- $\alpha .^{27,36)}$ Unexpectedly, the present study showed no significant differences in the levels of TNF- $\alpha$ in plasma $2 \mathrm{~h}$ after injection of endotoxin between endotoxintreated rats and endotoxin plus thalidomide-treated rats, suggesting that thalidomide does not inhibit endotoxin-induced overproduction of TNF- $\alpha$. The results obtained in this study are supported by the reports indicating that thalidomide did not inhibit endotoxin-induced overproduction of TNF- $\alpha$ in plasma and liver of rats, ${ }^{37)}$ and that it did not suppress TNF- $\alpha$ production in humans treated with endotoxin. ${ }^{38)}$ Therefore, these results at least suggest that TNF- $\alpha$ is not involved in the enhancement of endotoxin-induced decreases in the activity and expression of CYP3A2 by thalidomide. It is also reported that IL-1 $\beta$, IL- 2 and/or IL-6 play an important role in the down-regulation of hepatic CYP isoforms induced by endotoxin. ${ }^{2,39-42)}$ Fernández-Martínez et al. ${ }^{37)}$ described that thalidomide had no inhibitory effects on endotoxin-induced overproduction of IL- $1 \beta$ and IL- 6 . We cannot exclude the possibility that a sufficient concentration to inhibit endotoxin-induced overproduction of cytokines was not obtained by the dose of thalidomide used in this study. Further studies on concentration-inhibitory activity relationship of thalidomide are needed.

In conclusion, the present study suggests that thalidomide enhances endotoxin-induced decreases in the activity and function of the hepatic drug-metabolizing enzyme CYP3A2. It is unlikely that TNF- $\alpha$ and NO are involved in the enhancement of endotoxin-induced decreases in the activity and expression of hepatic CYP3A2 by thalidomide.

Acknowledgements This work was supported by a Grant-in-Aid for Scientific Research from the Ministry of Education, Culture, Sports, Science and Technology of Japan and the Scientific Research Promotion Fund from the Promotion and Mutual Aid Corporation for Private Schools of Japan.

\section{REFERENCES}

1) Minamiyama Y., Takemura S., Imaoka S., Funae Y., Tanimoto Y., Inoue M., Pharmacol. Exp. Ther, 283, 1479-1485 (1997).

2) Sewer M. B., Morgan E. T., Biochem. Pharmacol., 54, 729-737 (1997).

3) Morgan E. T., Li-Masters T., Cheng P. Y., Toxicology, 181-182, $207-210$ (2002).

4) Ueyama J., Kitaichi K., Nadai M., Iwase M., Tomyo N., Kanazawa H., Suzuki R., Takagi K., Takagi K., Hasegawa T., Eur. J. Pharmacol., 498, 257-265 (2004).

5) Ueyama J., Nadai M., Kanazawa H., Iwase M., Nakayama H., Hashimoto K., Yokoi T., Baba K., Takagi K., Takagi K., Hasegawa T. Eur. J. Pharmacol., 510, 127-134 (2005).

6) Hara H., Adachi T., Mol. Pharmacol., 61, 194-200 (2002).

7) Delaporte E., Renton K. W., Life Sci., 60, 787-796 (1997).

8) Iber H., Chen Q., Cheng P. Y., Morgan E. T., Arch. Biochem. Biophys., 377, 187-194 (2000).

9) Warren G. W., van Ess P. J., Watson A. M., Mattson M. P., Blouin R. A., J. Interferon Cytokine Res., 21, 821-826 (2001).
10) Beigneux A. P., Moser A. H., Shigenaga J. K., Grunfeld C., Feingold K. R., Biochem. Biophys. Res. Commun., 293, 145-149 (2002).

11) D'Amato R. J., Loughnan M. S., Flynn E., Folkman J., Proc. Natl. Acad. Sci. U.S.A., 91, 4082-4085 (1994).

12) Yabu T., Tomimoto H., Taguchi Y., Yamaoka S., Igarashi Y., Okazaki T., Blood, 106, 125-134 (2005).

13) Wettstein A. R., Meagher A. P., Lancet, 350, 1445-1446 (1997).

14) Marriott J. B., Muller G., Dalgleish A. G., Immunol. Today, 20, 538 540 (1999).

15) Hideshima T., Chauhan D., Shima Y., Raje N., Davies F. E., Tai Y. T., Treon S. P., Lin B., Schlossman R. L., Richardson P., Muller G., Stirling D. I., Anderson K. C., Blood, 96, 2943-2950 (2000).

16) Teo S. K., Stirling D. I., Zeldis J. B., Drug Discov. Today, 10, 107 114 (2005).

17) Hashimoto Y., Bioorg. Med. Chem., 10, 461-479 (2002).

18) Hashimoto Y., Mini Rev. Med. Chem., 2, 543-551 (2002).

19) Bartlett J. B., Dredge K., Dalgleish A. G., Nature Rev. Cancer, 4, 314-322 (2004).

20) Kim Y. S., Kim J. S., Jung H. C., Song I. S., Mol. Cells, 17, 210-216 (2004).

21) Zhang Z. L., Liu Z. S., Sun Q., World J. Gastroenterol., 11, 216-220 (2005).

22) Enomoto N., Takei Y., Hirose M., Ikejima K., Miwa H., Kitamura T., Sato N., Gastroenterology, 123, 291-300 (2002).

23) Enomoto N., Takei Y., Hirose M., Kitamura T., Ikejima K., Sato N., Alcohol Clin. Exp. Res., 27, 2S-6S (2003).

24) Schmidt H., Rush B., Simonian G., Murphy T., Hsieh J., Condon M., J. Surg. Res., 63, 143-146 (1996).

25) Giamarellos-Bourboulis E. J., Poulaki H., Kostomitsopoulos N., Dontas I., Perrea D., Karayannacos P. E., Giamarellou H., Antimicrob. Agents Chemother, 47, 2445-2449 (2003).

26) Shimazawa R., Sano H., Tanatani A., Miyachi H., Hashimoto Y., Chem. Pharm. Bull., 52, 498-499 (2004).

27) Enomoto N., Takei Y., Hirose M., Ikejima K., Kitamura T., Sato N., Comp. Hepatol., 3, S37 (2004).

28) Soucek P., Gut I., Xenobiotica, 22, 83-103 (1992)

29) Nadai M., Sekido T., Matsuda I., Wang L., Kitaichi K., Itoh A., Nabeshima T., Hasegawa T., J. Pharm. Pharmacol., 50, 871-879 (1998).

30) Omura T., Sato R., J. Biol. Chem., 239, 2370-2378 (1964).

31) Moreira A. L., Sampaio E. P., Zmuidzinas A., Frindt P., Smith K. A., Kaplan G., J. Exp. Med., 177, 1675-1680 (1993).

32) Waxman D. J., Biochem. Pharmacol., 37, $71-84$ (1988).

33) Sewer M. B., Morgan E. T., J. Pharmacol. Exp. Ther, 287, 352-358 (1998).

34) Takemura S., Minamiyama Y., Imaoka S., Funae Y., Hirohashi K., Inoue M., J. Hepatol., 30, 1035-1044 (1999).

35) Sampaio E. P., Sarno E. N., Galilly R., Cohn Z. A., Kaplan G., J. Exp. Med., 173, 699-703 (1991).

36) Watanabe N., Suzuki J., Kobayashi Y., J. Biochem. (Tokyo), 120, 1190-1195 (1996).

37) Fernández-Martínez E., Morales-Ríos M. S., Pérez-Álvarez V., Muriel P., Biochem. Pharmacol., 68, 1321-1329 (2004).

38) Shannon E., Noveck R., Sandoval F., Kamath B., Kearney M., Transl. Res., 150, 275-280 (2007).

39) Chen Y. L., Florentin I., Batt A. M., Ferrari L., Giroud J. P., ChauvelotMoachon L., Biochem. Pharmacol., 44, 137-148 (1992).

40) Morgan E. T., Thomas K. B., Swanson R., Vales T., Hwang J., Wrigh K., Biochim. Biophys. Acta, 1219, 475-483 (1994).

41) Tinel M., Elkahwaji J., Robin M. A., Fardel N., Descatoire V., Haouzi D., Berson A., Pessayre D., J. Pharmacol. Exp. Ther., 289, 649-655 (1999).

42) Iber H., Li-Masters T., Chen Q., Yu S., Morgan E. T., J. Pharmacol. Exp. Ther, 297, 174-180 (2001). 